\title{
Canopy Dynamics in Nanoscale Ionic Materials
}

\author{
Michael L. Jespersen, ${ }^{1,2}$ Peter A. Mirau, ${ }^{1 *}$ Ernst von Meerwall, ${ }^{3}$ Richard A. Vaia, ${ }^{1}$ Robert Rodriguez, ${ }^{4}$ \\ and Emmanuel P. Giannelis ${ }^{4}$
}

Air Force Research Laboratories, Materials and Manufacturing Directorate, Wright-Patterson AFB, Ohio 45433

peter.mirau@wpafb.af.mil

\begin{abstract}
RECEIVED DATE (to be automatically inserted after your manuscript is accepted if required according to the journal to which you are submitting your paper)
\end{abstract}

${ }^{1}$ Air Force Research Laboratories, Materials and Manufacturing Directorate (AFRL/RXBN), WrightPatterson AFB, OH 45433

${ }^{2}$ National Research Council, 500 Fifth St. NW, Keck 568, Washington, DC, 20001

${ }^{3}$ Department of Physics, University of Akron, Akron, OH 44325

${ }^{4}$ Department of Materials Science and Engineering, Cornell University, Ithaca, NY 14853

Abstract: Nanoscale ionic materials (NIMS) are organic-inorganic hybrids in which a core nanostructure is functionalized with a covalently attached corona and an ionically tethered organic canopy. NIMS are engineered to be liquids under ambient conditions in the absence of solvent and are of interest for a variety of applications. We have used nuclear magnetic resonance (NMR) relaxation and pulse-field gradient (PFG) diffusion experiments to measure the canopy dynamics of NIMS prepared from 18-nm silica cores modified by an alkylsilane monolayer possessing terminal sulfonic 
acid functionality, paired with an amine-terminated ethylene oxide/propylene oxide block copolymer canopy. Carbon NMR studies show that the block copolymer canopy is mobile both in the bulk and in the NIMS, and that the fast (ns) dynamics are insensitive to the presence of the silica nanoparticles. Canopy diffusion in the NIMS is slowed relative to the neat canopy, but not to the degree predicted from the diffusion of hard-sphere particles. Canopy diffusion is not restricted to the surface of the nanoparticles and shows unexpected behavior upon addition of excess canopy. Taken together, these data indicate that the liquid-like behavior in NIMS is due to rapid exchange of the block copolymer canopy between the ionically modified nanoparticles.

KEYWORDS: nanoscale ionic materials, nanoparticle liquids, ionic liquids, nuclear magnetic resonance, silica nanoparticles

Nanoscale ionic materials (NIMS) are an emerging class of functionalized nanoparticles that have generated a great deal of interest in recent years as a consequence of their unique materials properties. ${ }^{1-2}$ NIMs are organic-inorganic hybrids consisting of a core inorganic nanostructure functionalized with a covalently-bound organic corona with ionic terminal functionality and an ionically-tethered, bulky counter-ion as the canopy (Figure 1). In essence, NIMS are nanoparticles modified with an ionic liquid organic coating and either behave as liquids at room temperature or undergo reversible, macroscopic solid-to-liquid transitions near room temperature. To date, a number of NIMS have been prepared, including those based on metal oxides $\left(\mathrm{SiO}_{2},{ }^{2-4} \mathrm{Fe}_{2} \mathrm{O}_{3},{ }^{4} \mathrm{TiO}_{2},{ }^{1}\right.$ and $\left.\mathrm{ZnO}^{5}\right)$ and metals $\left(\mathrm{Au},{ }^{6-8} \mathrm{Pt}^{6-7}, \mathrm{Pd},{ }^{6}\right.$ and $\mathrm{Rh}^{6}$ ). This materials design motif has also been used to create room temperature liquids from other nanoscale materials, including carbon nanotubes ${ }^{9-10}$ and proteins. ${ }^{11}$ 


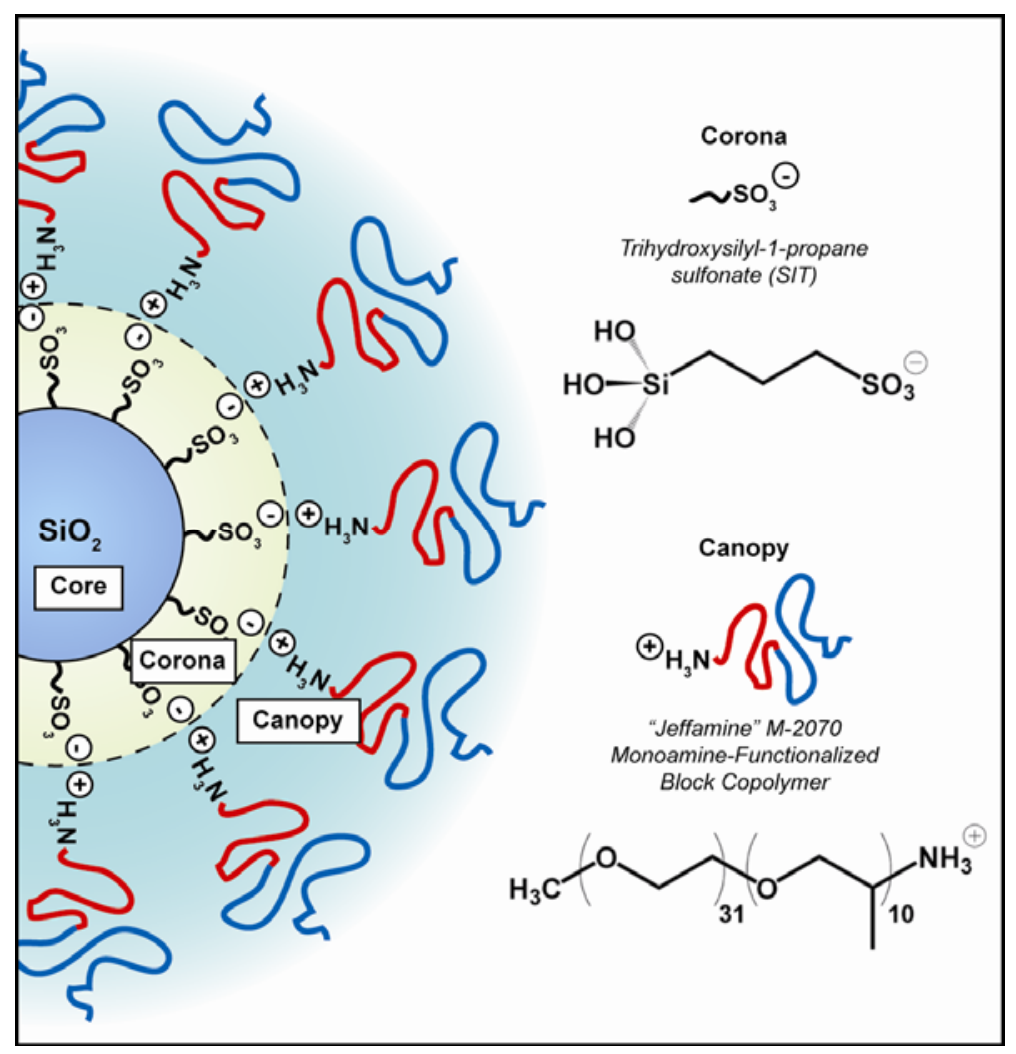

Figure 1. Schematic illustration of the general structure for nanoscale ionic materials (NIMS) used in this study. In this case, the covalently attached corona has terminal sulfonic acid functionality. The canopy consists of a low molecular weight, amine-terminated diblock copolymer.

The ability of NIMS to behave as room temperature liquids allows for the design of materials that combine characteristics of liquids with the unique size-dependent properties of the core nanostructures (e.g., photoluminescence with high viscosity, ${ }^{5}$ etc.). Exploiting the unique properties of NIMS in a broad range of applications will ultimately depend on the degree to which their properties can be tuned based on variations in their structural components (core shape and size; corona length and composition; and canopy composition and molecular weight). The ability to tailor multiple components makes NIMS a particularly versatile class of nanomaterials that can be designed to meet the specific requirements of many diverse materials and device applications. Consequently, NIMS are promising candidates for thermal management materials, ${ }^{12-18}$ nanocomposite materials, novel reaction solvents, ${ }^{19-20}$ magnetic fluids, ${ }^{21-22}$ and conductive lubricants. ${ }^{6-8}$ For example, our laboratory has reported on Au- and Pt-based 
NIMS employed as conductive lubricants that markedly improved the durability of RF-MEMS contacts. $^{7-8}$ While further improvements in performance can likely be realized through systematic variation of NIMS composition, structure, and chemistry, it will also be necessary to understand the dynamics of the corona-canopy interface in order to take full advantage of the functional opportunities that exist for NIMS. To date, molecular-level dynamics in these materials has not been studied.

Molecular-level characterization of organic corona dynamics in nanoparticles remains a key challenge for which general characterization techniques are still required within the broader nanomaterials community. Comprehensive surface and interface analysis typically requires a combination of composition analysis (XPS, Auger, TOF-SIMS, EPMA, etc.), electron microscopy (TEM, SEM), and scanning probe microscopy (AFM, STM, etc.) techniques to characterize composition, size, morphology, and structural order in nanomaterials and their assemblies. ${ }^{23-26}$ In general, these characterization techniques are well-suited for static surfaces in which the composition and position of the nanostructures remains constant. In contrast, nanomaterial interfaces in solution, such as that between the canopy and the corona in NIMS, or between the covalently-bound corona and the nanoparticle core, are likely dynamic. Nuclear magnetic resonance spectroscopy (NMR) is frequently used to study molecular-level dynamics and diffusion in macromolecules and polymers, ${ }^{27-28}$ and these NMR techniques can be extended to characterize composition, dynamics, and diffusion in nanomaterials. $^{29-32}$ We reasoned that these techniques could be employed to characterize dynamics in NIMS.

Here we report NMR relaxation and diffusion studies of silica-based NIMS with a bulky organic canopy. The results show that the canopy is very mobile in the liquid state, even in the presence of the nanoparticles. This suggests that the canopy molecules in the NIMS undergo rapid exchange between nanoparticles, rather than simply undergoing hard-sphere translational diffusion. The canopy exists in a liquid-like state, rapidly exchanging on and off the functionalized core nanostructure, and plays a critical role in the liquid-like properties. 


\section{Results and Discussion}

In order to characterize the dynamics of the corona-canopy interface, the spin-lattic relaxation and diffusion of the bulk free canopy were compared to that of the NIMS by solution NMR relaxation and pulse-field gradient (PFG) NMR. The NIMS of interest consisted of an 18-nm silica nanoparticle core functionalized with a monolayer of trihydroxysilylpropyl sulfonic acid (SIT). The anionic SIT corona was paired with a cationic canopy consisting of a primary amine-terminated ethylene oxide/propylene oxide diblock copolymer $\left(\mathrm{M}-2070, \mathrm{M}_{\mathrm{n}}=2263, \mathrm{M}_{\mathrm{w}}=2334, \mathrm{PDI}=1.03\right)$ canopy (Figure 1). NIMS have been described as monolithic hybrid materials with liquid properties, where each nanostructure "carries"

its share of the solvent. ${ }^{2,33}$ For this reason, a canopy: corona ratio of $1: 1$ was chosen as the most reasonable starting point for examining the molecular-level dynamics of NIMS using NMR. Figure 2 compares the $125 \mathrm{MHz}$ carbon NMR spectra of the bulk, unbound M-2070 canopy and the canopy incorporated into the NIMS. The main features to note are the methyl peak at $21 \mathrm{ppm}$ from the propylene oxide and the peaks at $74 \mathrm{ppm}$ and $79 \mathrm{ppm}$ assigned to the methine and methylene carbons from propylene oxide and the methylene carbons from ethylene oxide. M-2070 contains approximately $73 \%$ ethlyene oxide, and we have used the ethylene oxide peak at $74 \mathrm{ppm}$ to measure the canopy dynamics. Resonances from the propyl carbons in the SIT corona are not observed because they are present at much lower concentrations than the M-2070 methylenes and are broadened due to restricted motion and their close proximity to the nanoparticle. 


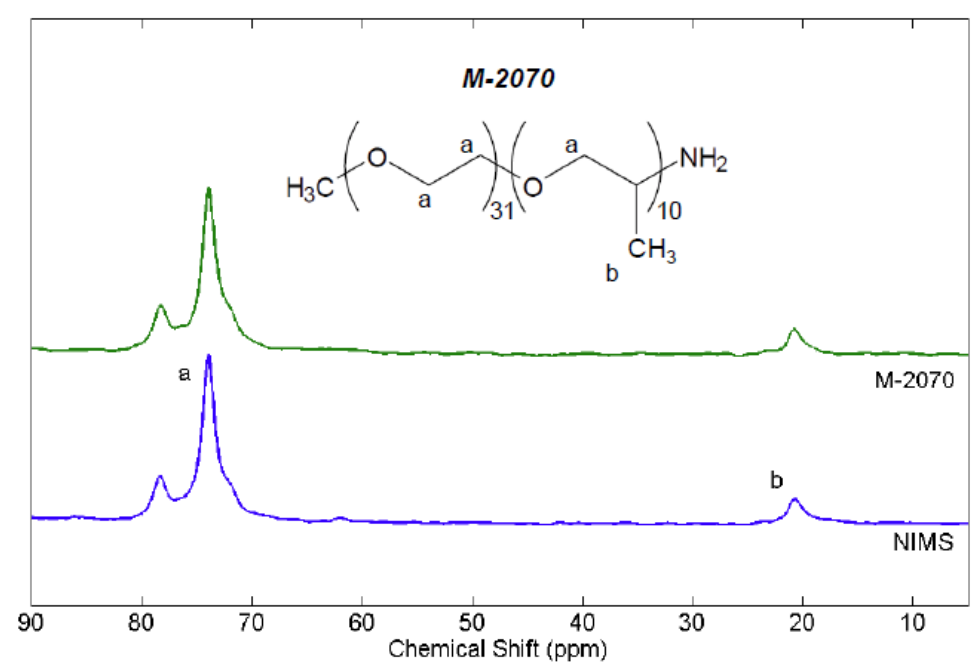

Figure 2. The $125 \mathrm{MHz}{ }^{13} \mathrm{C}$ NMR spectra of neat, dry M-2070 (top) and NIMS (bottom). The main features are the peaks at $74 \mathrm{ppm}$ and $21 \mathrm{ppm}$ assigned to the methylene and methine carbons in the ethylene oxide/propylene oxide and the methyl carbons in the propylene oxide, respectively.

$T_{1}$ Relaxation by Dipolar Interactions. The dynamics of the free canopy (M-2070) and the NIMS canopy in neat, dry samples were measured from the carbon spin-lattice relaxation times $\left(\mathrm{T}_{1}\right)$ of the methylene peak at $74 \mathrm{ppm}$. The spin-lattice relaxation time can be measured by determining the rate constant for the relaxation of a spin system to its equilibrium magnetization immediately following perturbation with a radio frequency pulse. $^{34}$ In addition, the $T_{1}$ relaxation times measured using observable magnetization can be used to extract information about fast (ns) molecular dynamics. This is because the spin-lattice relaxation rate is dominated by dipolar interactions of the carbon with its directly bonded protons and is given by ${ }^{35}$

$$
\frac{1}{T_{1}}=\frac{1}{10} \frac{\gamma_{C}^{2} \gamma_{H}^{2} \hbar^{2}}{r_{C H}^{6}}\left\{J\left(\omega_{C}-\omega_{H}\right)+3 J\left(\omega_{C}\right)+6 J\left(\omega_{H}+\omega_{C}\right)\right\}
$$

where $\gamma_{C}$ and $\gamma_{H}$ are the gyromagnetic ratios for carbon and proton nuclei, $r_{C H}$ is the $\mathrm{C}$ - $\mathrm{H}$ distance, and the spectral densities $J(\omega)$ are given by Fourier transform of the orientational autocorrelation function 
$(C(t))$ of the $\mathrm{C}-\mathrm{H}$ vector. The challenge in extracting molecular dynamics information from the $\mathrm{T}_{1}$ 's is that a number of models can often account for the relaxation data. ${ }^{27,36}$ The general strategy for fitting $T_{1}$ values is to measure the relaxation times as a function of temperature and magnetic field, then use the simplest model that fits the data. The models for chain dynamics in polymers include fast librational motion, slower segmental motion, and very slow Rouse-type motions. ${ }^{37-38}$ The canopy molecules of interest here are below or near the entanglement $\operatorname{limit}^{\dagger}$ and are intermediate between polymers and small molecules.

The first step in characterizing the dynamics by NMR relaxation is measurement of the $T_{1}$ relaxation across a range of temperatures and identification of the carbon $T_{1}$ minimum. The minimum occurs when the molecular motions are near the observation frequency $(125 \mathrm{MHz})$, and the depth and breadth of the $T_{1}$ versus temperature curve is sensitive to the rate and amplitude of molecular motions. Figure 3 compares the temperature dependence of the carbon $T_{1}$ 's for the bulk M-2070 and the NIMS canopy. The most remarkable feature of this plot is that the relaxation times (and molecular dynamics) of the ethylene oxide units do not seem to be sensitive to the presence of the 18-nm silica core.

\footnotetext{
${ }^{\dagger} \mathrm{M}_{\mathrm{e}}=1600-2200 \mathrm{~g} / \mathrm{mol}$ for PEO; $\mathrm{M}_{\mathrm{e}}=1287 \mathrm{~g} / \mathrm{mol}$ for PPO; $\mathrm{M}_{\mathrm{e}}=\mathrm{M}_{\mathrm{c}} / 2$
} 


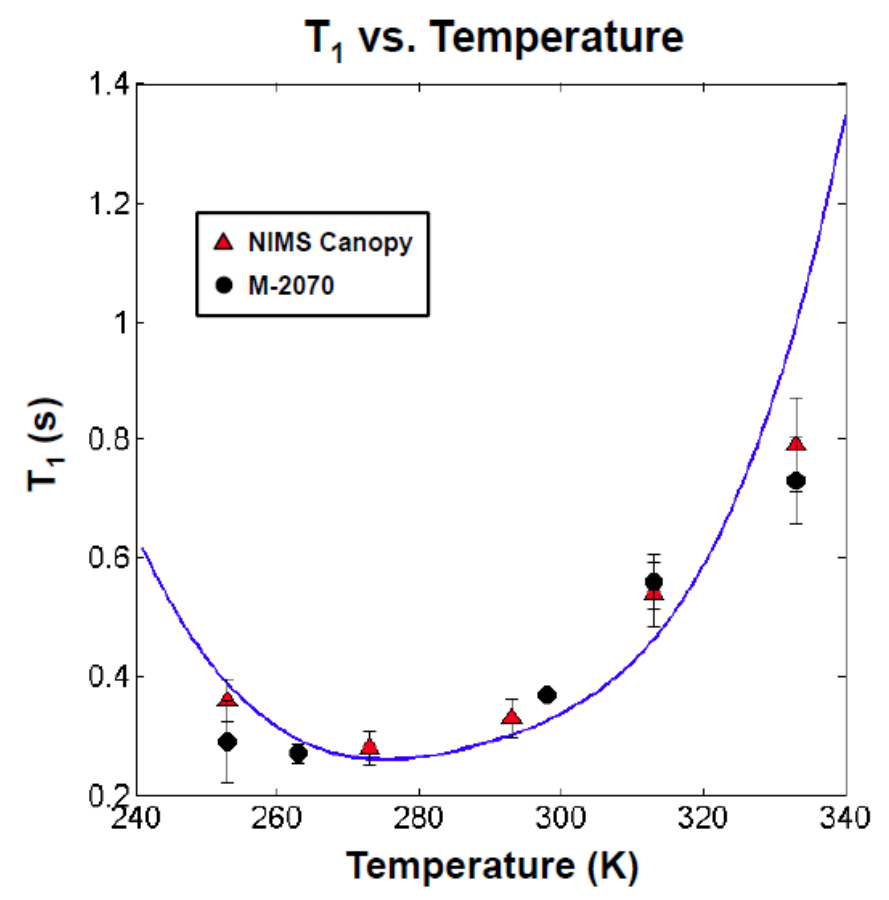

Figure 3. A plot of the relaxation times for the neat M-2070 and the NIMS canopy as a function of temperature. The solid line is calculated from the estimated correlation times as described in the Supporting Information.

A more quantitative understanding of canopy dynamics in NIMS can be obtained by fitting the temperature dependence of the relaxation to a specific model. A number of models for the $\mathrm{C}-\mathrm{H}$ autocorrelation function were explored to determine the rotational correlation times from the relaxation data. We observed that the $\mathrm{T}_{1}$ minimum $(0.1 \mathrm{~s})$ calculated from the isotropic reorientation model for the $\mathrm{C}-\mathrm{H}$ autocorrelation function did not adequately predict the experimentally observed $\mathrm{T}_{1}$ minimum $(0.26$ s) for either the neat canopy or the NIMS. Given that the canopy molecules are smaller than an entangled polymer in the melt, we have chosen to use the Lipari-Szabo (LS) model ${ }^{39-40}$ for the autocorrelation function rather than a more sophisticated model like the modified KWW model $^{37}$ commonly used for high molecular weight polymers. The key assumption of the LS model is that the overall molecular reorientation is decoupled from faster internal motions, and the autocorrelation function is given by the product of the correlation function for overall reorientation and the correlation function for internal motion. The LS model with a generalized order parameter of 0.4 gave a good fit to 
the $\mathrm{T}_{1}$ minimum and was used to calculate the correlation time as a function of temperature as described in the Supporting Information. The solid line in Figure 3 is the average relaxation time calculated from the correlation times for the bulk canopy and the NIMS. The key conclusions from the $\mathrm{T}_{1}$ fits are that the carbon relaxation is due to a combination of rapid (10 ps) librational motions and slower (0.1-2 ns) reorientation, and that the dynamics do not appear to depend on the presence of the 18-nm silica nanoparticle.

Pulse-Field Gradient NMR Studies. One of the key assumptions in NIMS design is that each functionalized nanoparticle carries its share of the solvent or canopy because the charge on the ionic terminal functionality of the corona is balanced by a strongly associated, oppositely-charged canopy. ${ }^{2,33}$ If the NIMS canopy were strongly associated with the nanoparticle surface, we would expect the free canopy and NIMS canopy to exhibit very different self-diffusion coefficients. In order to compare the diffusion behavior of M-2070 in the bulk and as the NIMS canopy, we utilized PFG NMR. The diffusion coefficients were measured using the stimulated-echo PFG pulse sequence (Figure 4) with corrections for gradient artifacts. ${ }^{41}$ In the case of a single diffusing species, the signal decay as a function of the gradient strength is given by ${ }^{42-43}$

$$
M(G)=M_{0} e^{-\gamma^{2} G^{2} \delta^{2} D(\Delta-\delta / 3)}
$$

where $\gamma$ is the gyromagnetic ratio of the nucleus of interest, $\delta$ is the time of the gradient pulse, $\Delta$ is the diffusion delay time in the pulse sequence, $G$ is the gradient strength, and the diffusion coefficient $D$ is related to the hydrodynamic radius $r_{H}$ by ${ }^{43-44}$

$$
D=\frac{k_{B} T}{6 \pi \eta r_{H}}
$$

In those cases where single-exponential decay is not observed (vide infra), we have chosen to fit the data to a stretched exponential function, given by

$$
M(G)=M_{0} e^{-\left[\gamma^{2} G^{2} \delta^{2} D(\Delta-\delta / 3)\right]^{\beta}}
$$




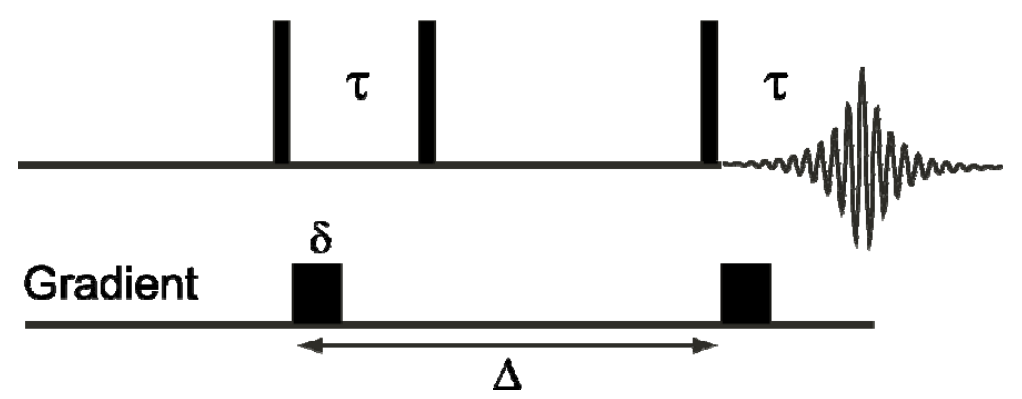

Figure 4. The pulse sequence diagram for the stimulated-echo experiment used for self-diffusion measurements.

The first measure of the interaction between the anionic terminal functionality on the nanoparticle and the cationic corona is to compare the diffusion behavior of solutions of the canopy and NIMS. Figure 5 compares the diffusion curves for $8 \%(w / w)$ solutions of the canopy and the NIMS in $\mathrm{D}_{2} \mathrm{O}$ at $50^{\circ} \mathrm{C}$. The most notable feature is that the diffusion coefficients are very similar for the two samples. In both cases, the data was fit to a stretched exponential function as given by Equation 4, and the results are listed in Table 1. 


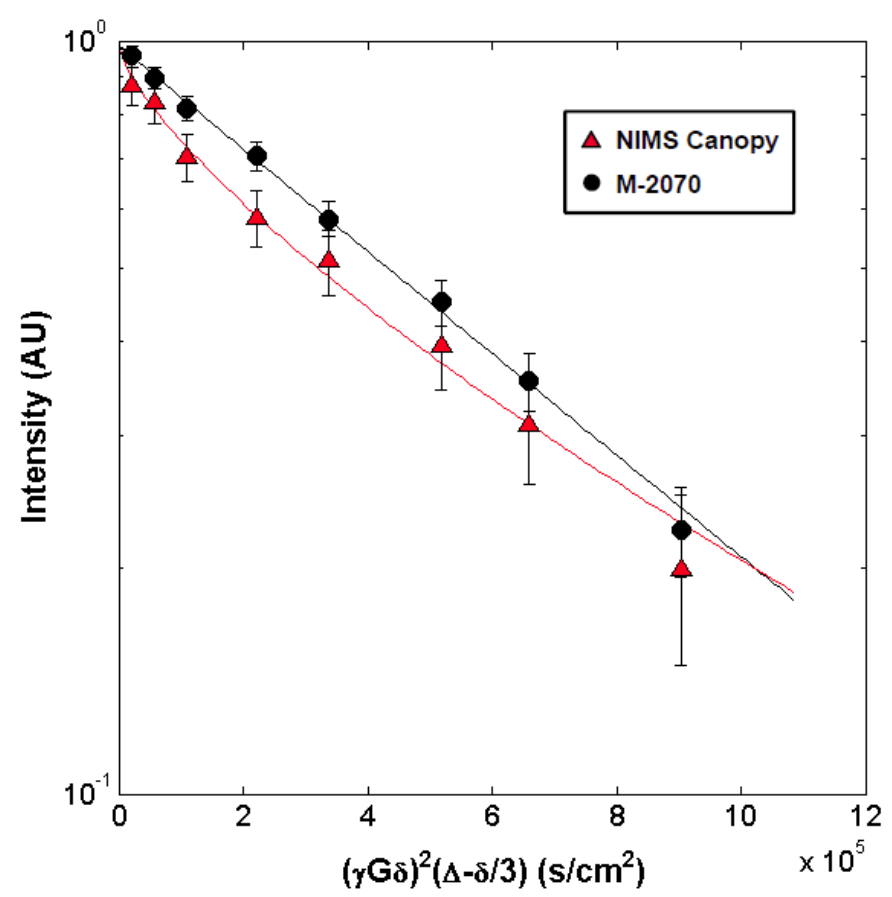

Figure 5. The PFG diffusion decay for $\mathrm{M}-2070\left(8 \% \mathrm{w} / \mathrm{w}\right.$ in $\left.\mathrm{D}_{2} \mathrm{O}\right)$ and as the NIMS canopy $(8 \% \mathrm{w} / \mathrm{w}$ in $\left.\mathrm{D}_{2} \mathrm{O}\right)$ at $50^{\circ} \mathrm{C}$.

Table 1. Self-diffusion coefficients and fitting parameters for the canopy and NIMS in $\mathrm{D}_{2} \mathrm{O}$ at $50^{\circ} \mathrm{C}$.

\begin{tabular}{lcc}
\hline Sample & $\boldsymbol{D}\left(\mathbf{c m}^{2} / \mathbf{s}\right)$ & $\boldsymbol{\beta}$ \\
\hline $\mathrm{M}-2070\left(\right.$ in $\left.\mathrm{D}_{2} \mathrm{O}\right)$ & $1.84 \times 10^{-6}$ & 0.98 \\
NIMS (in $\left.\mathrm{D}_{2} \mathrm{O}\right)$ & $1.56 \times 10^{-6}$ & 0.73 \\
\hline
\end{tabular}

The observable magnetization for $\mathrm{M}-2070$ in $\mathrm{D}_{2} \mathrm{O}$ appears to exhibit single exponential decay $(\beta=0.98)$ within experimental error, as expected for a monodisperse polymer $(\mathrm{PDI}=1.06)^{45}$ The measured diffusion coefficient for M-2070 shown in Table $1\left(1.84 \times 10^{-6} \mathrm{~cm}^{2} / \mathrm{s}\right)$ is in close agreement with the value of $2.36 \times 10^{-6} \mathrm{~cm}^{2} / \mathrm{s}$ calculated for $\mathrm{M}-2070$ in $\mathrm{D}_{2} \mathrm{O}$ at $50^{\circ} \mathrm{C}$, based upon an estimated hydrodynamic radius of $1.25 \mathrm{~nm}$, assuming a freely jointed chain. ${ }^{46}$ This estimated hydrodynamic radius is similar to that determined from dynamic light scattering experiments (not shown), which 
showed a hydrodynamic radius of $1.15 \mathrm{~nm}$ for M-2070. By comparison, the decay curve for the NIMS canopy shows only a $15 \%$ decrease in the diffusion coefficient compared to dissolved M-2070. We can estimate the diffusion coefficient expected if the canopy molecules were bound to the nanoparticles by calculating the hydrodynamic radius for the silica nanoparticle $(9 \mathrm{~nm})$, the corona $(0.5 \mathrm{~nm})$ and the canopy $(2 \times 1.25 \mathrm{~nm})$. This gives a hard-sphere radius of $12 \mathrm{~nm}$, so we would expect the diffusion coefficient to decrease by a factor of 9.6 relative to a dissolved canopy molecule in $\mathrm{D}_{2} \mathrm{O}$. The fact that we observe only a $15 \%$ decrease in the diffusion coefficient shows that the NIMS canopy does not undergo hard-sphere diffusion in solution. Rather, the canopy is exchanging between free molecules and other nanoparticles in $\mathrm{D}_{2} \mathrm{O}$ on the time scale $(\mathrm{ms})$ of the diffusion measurements.

We also note that curvature is observed in the NIMS self-diffusion data, requiring the use of a stretched exponential or some other complex function for data analysis. This curvature arises from a distribution of diffusion coefficients reflecting the statistical nature of the ionic interactions between the canopy and the corona. This distribution arises from the mismatch between the effective "footprint" of the canopy molecule $\left(\sim 4.9 \mathrm{~nm}^{2}\right)$ and that of a single SIT molecule bound to the nanoparticle surface $\left(\sim 0.2 \mathrm{~nm}^{2}\right)$. This size mismatch makes it impossible for all of the canopy molecules to be in direct contact with the corona and suggests that several layers of canopy molecules are required to neutralize the SIT molecules bound to the nanoparticle core. The electrostatic interaction between the canopy and corona will be strongest at the particle surface and weakest at the outermost layers of canopy far from the particle, giving rise to a distribution in exchange rates and a distribution in diffusion coefficients observed in the pulsed-field gradient diffusion experiments. This phenomenon has been suggested by previous work from our laboratory (MacCuspie, R. I.; Elsen, A. M.; Diamanti, S. J.; Patton, S. T.; Altfeder, I.; Jacobs, J. D.; Voevodin, A. A.; Vaia, R. A. Previously unpublished work.) and seems to be a reasonable source for the observed deviation from single exponential behavior in the NIMS selfdiffusion data. Furthermore, we would expect weakly-associated canopy in the diffuse layer around the 
nanoparticle to exchange more readily than the canopy molecules more closely associated with the surface-bound corona.

Figure 6 compares the self-diffusion decay curves for neat, dry samples of the free canopy, NIMS, and NIMS spiked with a two-fold excess of M-2070 relative to the number of anionic sites on the nanoparticle. In all cases we observe curvature on the semilog plots and good fits to the stretched exponential function. The diffusion coefficients and fit parameters are listed in Table 2.

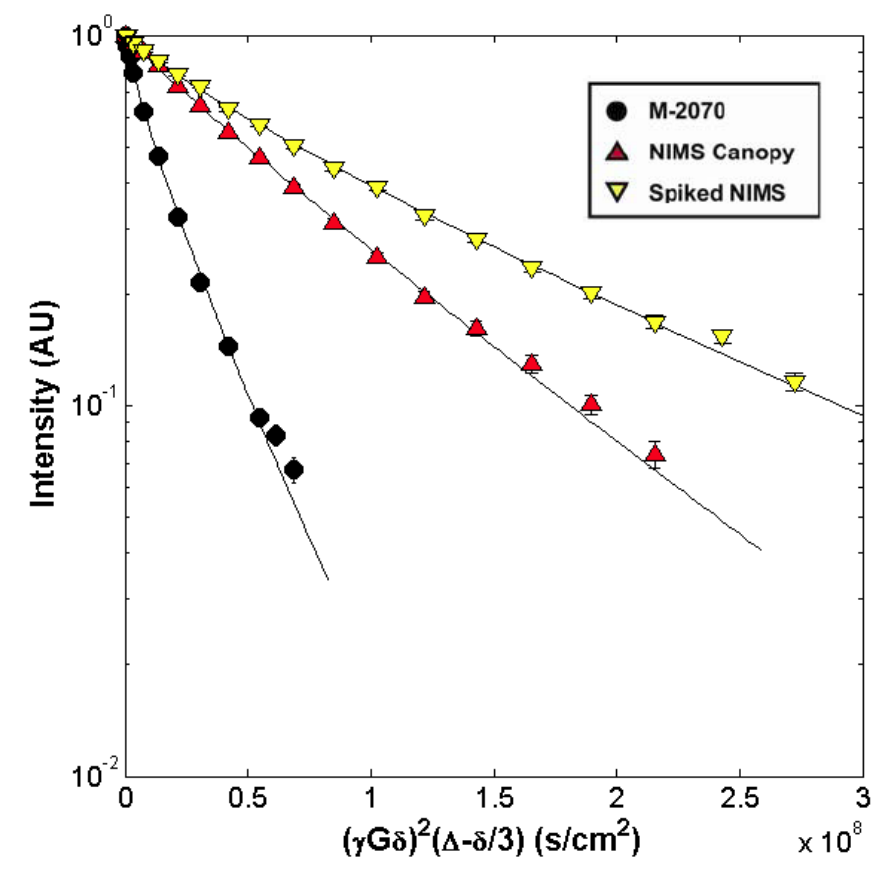

Figure 6. The self-diffusion decay curves for the neat, dry samples of bulk canopy, NIMS canopy, and NIMS spiked with a two-fold excess of M-2070 at $50^{\circ} \mathrm{C}$.

Even though we observed single exponential decay for M-2070 in $\mathrm{D}_{2} \mathrm{O}$ solution, Figure 6 and Table 2 show that a stretched exponential $(\beta=0.83)$ is required to fit the data for the neat canopy. Since the solution experiments eliminate polydispersity as an explanation for this curvature in the signal decay, we believe the curvature results from self-association of M-2070 in the neat state, which does not occur when the molecule is dissolved in $\mathrm{D}_{2} \mathrm{O}$ (Figure 5). We note that $\mathrm{M}-2070$ is similar in structure to the Pluronic block copolymers, which are known to self-associate in solution and give rise to 
distributions in diffusion coefficients. ${ }^{47}$ We have also observed self-association of M-2070 in GPC and small angle x-ray scattering experiments (not shown).

Table 2. Self diffusion coefficients for neat M-2070, NIMS canopy, and NIMS with a two-fold excess of M-2070 relative to the number of anionic sites on the nanoparticle.

\begin{tabular}{lccc}
\hline Sample & $\boldsymbol{\Delta ( \mathbf { m s } )}$ & $\boldsymbol{D}\left(\mathbf{c m}^{2} \mathbf{s}\right)$ & $\boldsymbol{\beta}$ \\
\hline Bulk M-2070 & 70 & $5.3 \times 10^{-8}$ & 0.83 \\
& 500 & $5.4 \times 10^{-8}$ & 0.90 \\
NIMS Canopy & 70 & $1.2 \times 10^{-8}$ & 0.91 \\
& 300 & $1.3 \times 10^{-8}$ & 0.93 \\
NIMS Canopy + & 70 & $0.9 \times 10^{-8}$ & 0.84 \\
Excess M-2070 & & & \\
\hline
\end{tabular}

The data in Table 2 and Figure 6 also show that canopy diffusion in the bulk NIMS is slower by a factor of 4.5 than in the bulk M-2070, but this is not as slow as the factor of 9.6 predicted by the hardsphere model. We believe the faster-than-expected diffusion in the NIMS is the result of canopy exchange between nanoparticles, which is also a consequence of crowding at the nanoparticle surface and the subsequent weakening of electrostatic interaction between SIT and the outer canopy layers. It is well known that water contamination can affect the properties of ionic liquids, ${ }^{48}$ but we do not believe water has affected our results since we were unable to detect residual water by ${ }^{1} \mathrm{H}$ NMR in our dried samples at the 1:1 corona:canopy stoichiometry.

The canopy exchange model is further supported by the diffusion experiments where we spiked the NIMS sample with excess canopy. As shown in Figure 6 and Table 2, spiking leads to a decrease (30\%) in the diffusion coefficient and a broadening of the diffusion coefficient distribution $(\beta=0.84)$. This 
observation is inconsistent with the hard-sphere model, for which we would expect a biexponential decay curve, where $50 \%$ of the decay would be associated with the diffusion coefficient of bulk M-2070 and $50 \%$ with the diffusion coefficient of the hard-sphere particle. These data are also inconsistent with a model in which we have rapid exchange between the canopy molecules in all layers, in which case we would observe a signal decay curve intermediate between those measured for the NIMS and M-2070. The observation that the diffusion coefficient decreases in the spiked sample was unexpected, but we believe that this observation also results from crowding at the nanoparticle surface and a distribution of exchange rates between the canopy layers at varying distances from the nanoparticle surface. Molecular dynamics simulations are currently underway to clarify this behavior.

Another aspect of the dynamic behavior to consider in NIMS is the possibility of restricted diffusion of the canopy along the surface of the nanoparticle. Isotropic and restricted diffusion can be distinguished by comparing the diffusion following long and short diffusion delay times $(\Delta)$ in the stimulated-echo pulse sequence. ${ }^{42}$ In the case of free diffusion, we can calculate the translational displacement of a species during the delay time as ${ }^{46}$

$$
x=2 \sqrt{D \Delta}
$$

For a typical delay time in the stimulated echo pulse sequence $(70 \mathrm{~ms})$, this gives a translational displacement of $580 \mathrm{~nm}$ for the NIMS, a distance much greater than the perimeter of the nanoparticle. For a freely diffusing molecule, the diffusion coefficient will not depend on the delay time $\Delta$ in the PFG pulse sequence, while the apparent diffusion coefficient will decrease as the delay time $\Delta$ increases in molecules undergoing restricted diffusion. Figure 7 compares the diffusion plots for the NIMS with diffusion delay times of 70 and $300 \mathrm{~ms}$. The two plots overlap, indicating that canopy diffusion is not restricted to the nanoparticle surface in the NIMS. Unrestricted diffusion is also observed for bulk M2070 (Table 2). These results further support our model in which the canopy molecules exchange between nanoparticles. 


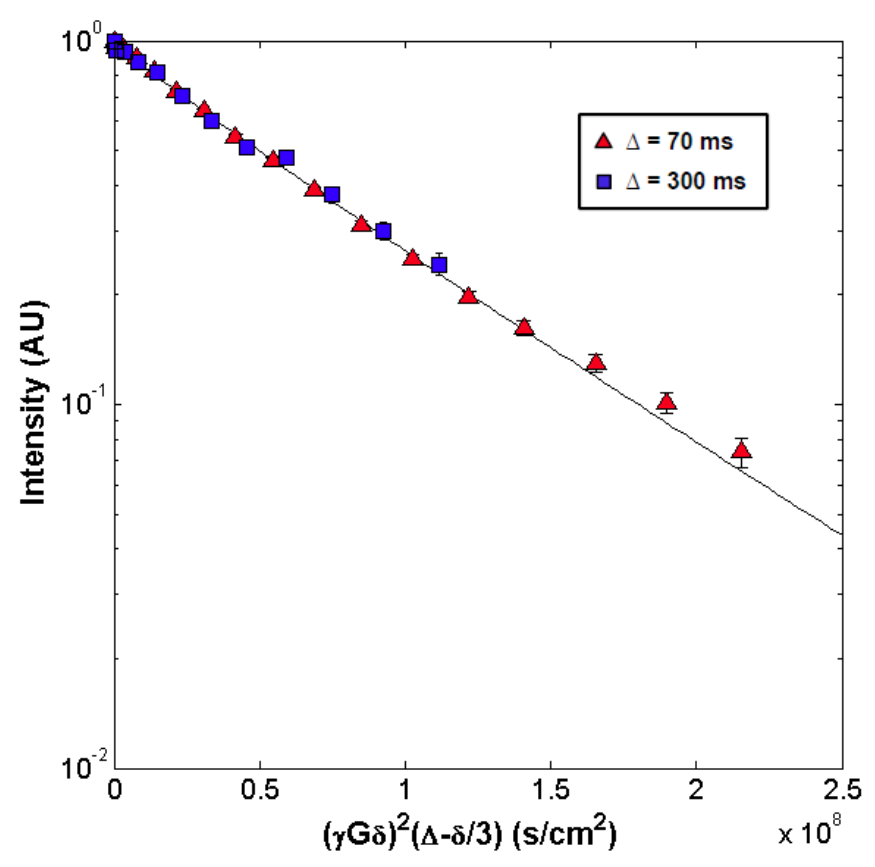

Figure 7. PFG diffusion plots for NIMS at $50^{\circ} \mathrm{C}$ with diffusion delay times of $\Delta=0.07 \mathrm{~s}$ and $\Delta=0.3 \mathrm{~s}$.

In summary, we have studied the dynamics and diffusion of the organic canopy in $\mathrm{SiO}_{2} \mathrm{NIMS}$ using a combination of NMR relaxation and pulse-field gradient techniques. The $\mathrm{T}_{1}$ relaxation times for the methylene carbons in the free canopy were measured as a function of temperature and were similar to those measured for the bound canopy in the NIMS, suggesting that the presence of the 18-nm silica core does not impact the fast (ns) dynamics of the canopy. Canopy diffusion in the NIMS was slowed relative to bulk canopy, but not to the extent expected for hard-sphere diffusion, suggesting that canopy molecules undergo exchange between nanoparticles. This hypothesis is supported by experimental results showing a single diffusion coefficient for the NIMS spiked with excess canopy, along with the observation that canopy diffusion is unrestricted in the NIMS. While the corona-canopy interface is often considered to be static, with each nanoparticle carrying its share of the liquid medium (or canopy), ${ }^{2,33}$ these results suggest that the corona-canopy interface is dynamic. We are currently investigating the impact of corona:canopy ratio, canopy molecular weight and the size and chemical structure of the nanoparticle core on the relaxation and diffusion characteristics of other nanoparticlebased ionic materials with liquid-like behavior at ambient temperature. 


\section{Methods}

Materials. Silica nanoparticles (LUDOX ${ }^{\circledR}$ HS-30 colloidal silica, 30 wt. \% suspension in $\mathrm{H}_{2} \mathrm{O}, 18$ nm diameter) were obtained and used as received from Aldrich Chemical Company (Milwaukee, WI). 3-(Trihydroxysilyl)-1-propanesulfonic acid (30-35 wt. \% solution in $\mathrm{H}_{2} \mathrm{O}$ ) was obtained and used as received from Gelest, Inc. (Morrisville, PA). Jeffamine ${ }^{\circledR}$ M-2070 Polyetheramine was obtained and used as received from Huntsman Corporation (The Woodlands, TX). Deuterium oxide (99.990 atom \% D) was obtained from Aldrich and used as received. Deionized water (18.2 M $\Omega-\mathrm{cm})$ was purified using a Barnstead Nanopure system.

Nanoparticle functionalization. Silica nanoparticles were functionalized according to a previously reported procedure. ${ }^{2}$ Briefly, colloidal silica (HS-30) was suspended in deionized water and diluted to a concentration of $3.75 \%$ by weight. At the same time, 3-(trihydroxysilyl)-1-propanesulfonic acid (SIT, 30-35 wt. $\%$ in deionized water) was diluted to a concentration of 8 wt. $\%$ using deionized water. The silica nanoparticle suspension was slowly added to the SIT solution dropwise under vigorous stirring. To this mixture, $1 \mathrm{M} \mathrm{NaOH}$ was slowly added until the solution $\mathrm{pH}$ was approximately 5 . The weakly acidic solution was then stirred vigorously for $24 \mathrm{hr}$ at $70{ }^{\circ} \mathrm{C}$. The functionalized nanoparticles were purified by removal of free SIT and ionic impurities through dialysis. The reaction solution was transferred into a dialysis membrane (Spectra/Por RC Biotech Membrane, 15K MWCO, $16 \mathrm{~mm}$ flat width) and dialyzed in deionized water for at least 48 hours prior to further surface modification. The functionalized nanoparticles were passed through an ion exchange column (HCR-W2 ion exchange resin, 20:1 ratio of resin to nanoparticles, by weight) three or four times to ensure complete replacement of $\mathrm{Na}^{+}$ions by protons. The $\mathrm{pH}$ of the nanoparticle solution following ion exchange is approximately 2.

NIMS Preparation. Once the SIT-functionalized $\mathrm{SiO}_{2}$ nanoparticles $\left(\mathrm{SIT}-\mathrm{SiO}_{2} \mathrm{NPs}\right)$ were purified and run through the ion exchange resin, a small sample was dried under vacuum and analyzed by thermogravimetric analysis (TGA) in order to determine the organic content and the extent of surface 
modification (usually $\sim 14-15$ wt. $\%$ organics). Based on this analysis, NIMS were prepared by addition of an appropriate amount of M-2070 in deionized water to the SIT-SiO 2 NP solution while stirring and monitoring the solution $\mathrm{pH}$. The equivalence point of the resulting titration curve indicates the acidic corona and weakly basic canopy are present in a 1:1 stoichiometric ratio. This ratio can be tuned to incorporate an excess or deficiency of canopy relative to a 1:1 corona:canopy ratio by adjusting the final $\mathrm{pH}$ of the solution. Once the appropriate amount of M-2070 had been added to generate the targeted corona:canopy ratio, the solvent was removed under vacuum at $35^{\circ} \mathrm{C}$ for several days, resulting in a light yellow, viscous product that flows as a liquid at room temperature.

NMR Sample Preparation. NIMS samples for NMR analysis were prepared by one of two methods, depending upon the experiment conducted. For initial collection of ${ }^{13} \mathrm{C}$ and ${ }^{1} \mathrm{H}$ spectra for peak assignments, a solution of $8 \%(\mathrm{w} / \mathrm{w}) \mathrm{M}-2070$ or $8 \%(\mathrm{w} / \mathrm{w})$ NIMS in $\mathrm{D}_{2} \mathrm{O}$ was prepared in a standard 5 mm NMR tube. Neat samples for relaxation and pulse-field gradient measurements were prepared by carefully transferring approximately $100 \mathrm{mg}$ neat M-2070 or neat NIMS to a 4-mm diameter NMR tube (cut to a length of approximately $1.5 \mathrm{~cm}$ ) inside an inert atmosphere glove box. Residual water was removed from the samples by heating to $30^{\circ} \mathrm{C}$ in a under vacuum for at least 48 hours. Each neat sample was then sealed with a commercially available epoxy resin, with a small plug of Teflon tape inserted into the tube between the sample and the epoxy in order to prevent undesired mixing. The epoxy seal was allowed to dry overnight under inert atmosphere.

Analytical Methods. ${ }^{13} \mathrm{C}$ relaxation experiments were conducted using a Tecmag Apollo $500 \mathrm{MHz}$ NMR spectrometer equipped with a DOTY Scientific DSI-1119 MAS probe and a DOTY Scientific temperature controller. Carbon $T_{1}$ relaxation experiments were carried out using an inversion-recovery pulse sequence, and the methylene carbon resonance at $74 \mathrm{ppm}$ was utilized to determine the $T_{1}$ relaxation time. The following values were used as the variable delay times to allow for relaxation of the spin system prior to application of the observation pulse: $0.01,0.02,0.05,0.1,0.2,0.5,1,2$, and 5 
seconds. The data for each $\mathrm{T}_{1}$ experiment were plotted and fit to an exponential function using a program designed for MatLab in order to extract $T_{1}$ relaxation times.

Diffusion coefficients were measured at $50.5^{\circ} \mathrm{C}$ using proton pulsed-gradient spin-echo NMR (PGSE NMR). This technique is based on pulsed nuclear magnetic resonance; the details of our implementation of PGSE NMR have been described previously. ${ }^{49}$ To summarize, the $33 \mathrm{MHz}$ SpinLock CPS-2 spectrometer was employed to produce a stimulated echo following the last $90^{\circ}$ pulse in a three-pulse radio-frequency sequence. The sequence was coordinated with a matched pair of pulsed magnetic field gradients of magnitude $\mathrm{G}=652 \mathrm{Gauss} / \mathrm{cm}$, separated by $\Delta=70 \mathrm{~ms}$. The spacing between the first two $90^{\circ}$ pulses was set to $15 \mathrm{~ms}$. To test for the possibility of restricted diffusion, additional measurements at $\Delta=300 \mathrm{~ms}$ were conducted for several samples. A steady gradient of magnitude $\mathrm{G}_{\mathrm{o}}=0.3 \mathrm{Gauss} / \mathrm{cm}$ was also applied for experimental convenience by narrowing the echo signal. As a result of the gradient pulses, the spin echo height $\mathrm{A}$ in the sample is attenuated as the sample molecules diffuse. The gradient pulse duration $\delta$ was adjusted in 15 to 20 steps until the echo signal was reduced to the background noise level. Audio signals were obtained using single side-band radio-frequency phase sensitive detection offset by $3 \mathrm{kHz}$ from the reference frequency, followed by Hamming-windowed magnitude Fourier transforms integrated over the echo peak, corrected for magnitude baseline noise. Echo height measurements were averaged over 6 to 12 signal passes. Results were then analyzed off-line using the latest version of the Fortran code DIFUS5K. ${ }^{50}$ The echo attenuation is expected to follow the general form

$$
\frac{A(X)}{A(0)}=\sum_{i} w_{i} \exp \left[-\gamma^{2} D_{i} X\right]
$$

with $\sum_{i} w_{i}=1$, where $\gamma$ is the proton gyromagnetic ratio, and $X=\delta^{2} \mathrm{G}^{2}(\Delta-\delta / 3)+$ small correction terms in $\mathrm{G}^{\cdot} \mathrm{G}_{\mathrm{o}}$. 
Acknowledgment. Funding provided by the Air Force Office of Scientific Research is gratefully acknowledged. The diffusion portion of this work was supported by the National Science Foundation under Grant DMR 04 55117. This publication is based on work supported by Award No. KUS-C1-01802, made by King Abdullah University of Science and Technology (KAUST). This research was carried out while M. Jespersen was an NRC Postdoctoral Fellow at the Air Force Research Laboratories. The authors would like to thank H. Koerner and M. Tchoul for assistance with SAXS experiments and GPC experiments, respectively, as well as for productive discussions regarding this research.

Supporting Information Available: Discussion of approach used to calculate correlation times and resulting $T_{1}$ versus temperature curves based on the LS model. This material is available free of charge via the Internet at http://pubs.acs.org. 


\section{References}

1. Bourlinos, A. B.; Chowdhury, S. R.; Herrera, R.; Jiang, D. D.; Zhang, Q.; Archer, L. A.; Giannelis, E. P. Functionalized Nanostructures with Liquid-Like Behavior: Expanding the Gallery of Available Nanostructures. Adv. Funct. Mater. 2005, 15, 1285-1290.

2 Rodriguez, R.; Herrera, R.; Archer, L. A.; Giannelis, E. P. Nanoscale Ionic Materials. Adv. Mater. 2008, 20, 4353-4358.

3. Bourlinos, A. B.; Chowdhury, S. R.; Jiang, D. D.; An, Y.-U.; Zhang, Q.; Archer, L. A.; Giannelis, E. P. Layered Organosilicate Nanoparticles with Liquidlike Behavior. Small 2005, 1, 80-82.

4. Bourlinos, A. B.; Herrera, R.; Chalkias, N.; Jiang, D. D.; Zhang, Q.; Archer, L. A.; Giannelis, E. P. Surface-Functionalized Nanoparticles with Liquid-Like Behavior. Adv. Mater. 2005, 17, 234237.

5. Bourlinos, A. B.; Stassinopoulos, A.; Anglos, D.; Herrera, R.; Anastasiadis, S. H.; Petridis, D.; Giannelis, E. P. Functionalized ZnO Nanoparticles with Liquidlike Behavior and Their Photoluminescence Properties. Small 2006, 2, 513-516.

6. Warren, S. C.; Banholzer, M. J.; Slaughter, L. S.; Giannelis, E. P.; DiSalvo, F. J.; Wiesner, U. B. Generalized Route to Metal Nanoparticles with Liquid Behavior. J. Am. Chem. Soc. 2006, 128, 12074-12075.

7. Voevodin, A. A.; Vaia, R. A.; Patton, S. T.; Diamanti, S.; Pender, M.; Yoonessi, M.; Brubaker, J.; Hu, J.-J.; Sanders, J. H.; Phillips, B. S.; MacCuspie, R. I. Nanoparticle-Wetted Surfaces for Relays and Energy Transmission Contacts. Small 2007, 3, 1957-1963.

8. Patton, S. T. Nanoparticle Liquids for Surface Modification and Lubrication of MEMS Switch Contacts. J. Microelectromech. Syst. 2008, 17, 741-746.

9. Bourlinos, A. B.; Georgakilas, V.; Boukos, N.; Dallas, P.; Trapalis, C.; Giannelis, E. P. SiliconeFunctionalized Carbon Nanotubes for the Production of New Carbon-Based Fluids. Carbon 2007, 45, 1583-1595.

10. Bourlinos, A. B.; Georgakilas, V.; Tzitzios, V.; Boukos, N.; Herrera, R.; Giannelis, E. P. Functionalized Carbon Nanotubes: Synthesis of Meltable and Amphiphilic Derivatives. Small 2006, 2, 1188-1191.

11. Perriman, A. W.; Colfen, H.; Hughes, R. W.; Barrie, C. L.; Mann, S. Solvent-Free Protein Liquids and Liquid Crystals. Angew. Chem. Int. Ed. 2009, 48, 6242-6246.

12. Eastman, J. A.; Choi, S. U. S.; Li, S.; Yu, W.; Thompson, L. J. Anomalously Increased Effective Thermal Conductivities of Ethylene Glycol-Based Nanofluids Containing Copper Nanoparticles. Appl. Phys. Lett. 2001, 78, 718-720.

13. Jang, S. P.; Choi, S. U. S. Role of Brownian Motion in the Enhanced Thermal Conductivity of Nanofluids. Appl. Phys. Lett. 2004, 84, 4316-4318. 
14. Jang, S. P.; Choi, S. U. S. Effects of Various Parameters on Nanofluid Thermal Conductivity. J. Heat Transfer 2007, 129, 617-623.

15. Jwo, C.-S.; Teng, T.-P.; Chang, H. A Simple Model to Estimate Thermal Conductivity of Fluid with Acicular Nanoparticles. J. Alloys Compd. 2007, 434-435, 569-571.

16. Murshed, S. M. S.; Leong, K. C.; Yang, C. A Model for Predicting the Effective Thermal Conductivity of Nanoparticle-Fluid Suspensions. Int. J. Nanosci. 2006, 5, 23-33.

17. Wang, X.; Xu, X.; Choi, S. U. S. Thermal Conductivity of Nanoparticle-Fluid Mixture. J. Thermophys. Heat Transfer 1999, 13, 474-480.

18. Yang, Y.; Zhang, Z. G.; Grulke, E. A.; Anderson, W. B.; Wu, G. Heat Transfer Properties of Nanoparticle-in-Fluid Dispersions (Nanofluids) in Laminar Flow. Int. J. Heat Mass Transfer 2005, 48, 1107-1116.

19. Ma, Z.; Yu, J.; Dai, S. Preparation of Inorganic Materials Using Ionic Liquids. Adv. Mater. 2009, $21,1-25$.

20. Shin, J. Y.; Lee, B. S.; Jung, Y.; Kim, S. J.; Lee, S.-G. Palladium Nanoparticles Captured onto Spherical Silica Particles Using a Urea Cross-Linked Imidazolium Molecular Band. Chem. Commun. 2007, 5238-5240.

21. Morais, P. C.; Santos, J. G.; Silveira, L. B.; Gansau, C.; Buske, N.; Nunes, W. C.; Sinnecker, J. P. Susceptibility Investigation of the Nanoparticle Coating-Layer Effect on the Particle Interaction in Biocompatible Magnetic Fluids. J. Magn. Magn. Mater. 2004, 272-276, 2328-2329.

22. Park, S. I.; Lim, J. H.; Kim, J. H.; Yun, H. I.; Roh, J. S.; Kim, C. G.; Kim, C. O. Effects of Surfactant on Properties of Magnetic Fluids for Biomedical Application. Phys. Status Solidi B 2004, 241, 1662-1664.

23. Briggs, D.; Grant, J. T., Surface Analysis by Auger and X-Ray Electron Spectroscopy. IM Publications and Surface Spectra Limited: West Sussex, 2003.

24. Vickerman, J. C., Surface Analysis: The Principal Techniques. John Wiley \& Sons: New York, 1997.

25. O'Connor, D. J.; Sexton, B. A.; Smart, R. S. C., Surface Analysis Methods in Materials Science. 2nd ed.; Springer: Berlin, 2003.

26. Richman, E. K.; Hutchison, J. E. The Nanomaterial Characterization Bottleneck. ACS Nano 2009, 3, 2441-2446.

27. Mirau, P. A Practical Guide to the NMR of Polymers. John Wiley \& Sons: Hoboken, 2004.

28. Bovey, F. A.; Mirau, P. A. NMR of Polymers. Academic Press: San Diego, 1996.

29. Kohlmann, O.; Steinmetz, W. E.; Mao, X.-A.; Wuilfing, W. P.; Templeton, A. C.; Murray, R. W.; Johnson, C. S. NMR Diffusion, Relaxation and Spectroscopic Studies of Water Soluble Monolayer-Protected Gold Nanoclusters. J. Phys. Chem. B 2001, 105, 8801-8809. 
30. Fritzinger, B.; Moreels, I.; Lommens, P.; Koole, R.; Hens, Z.; Martins, J. C. In Situ Observation of Rapid Ligand Exchange in Colloidal Nanocrystal Suspensions Using Transfer Noe Nuclear Magnetic Resonance Spectroscopy. J. Am. Chem. Soc. 2009, 131, 3024-3032.

31. Donkers, R. L.; Song, Y.; Murray, R. W. Substituent Effects on the Exchange Dynamics of Ligands on 1.6-nm Diameter Gold Nanoparticles. Langmuir 2004, 20, 4703-4707.

32. Song, Y.; Murray, R. W. Dynamics and Extent of Ligand Exchange Depend on Electronic Charge of Metal Nanoparticles. J. Am. Chem. Soc. 2002, 124, 7096-7102.

33. Agarwal, P.; Qi, H.; Archer, L. A. The Ages in a Self-Suspended Nanoparticle Liquid. Nano Lett. 2010, 10, 111-115.

34. Friebolin, H. Basic One- and Two-Dimensional NMR Spectroscopy. Wiley-VCH: Weinheim, 1998.

35. Abragam, A. Principles of Nuclear Magnetism. Oxford University Press: New York, 1961.

36. Bovey, F. A. Nuclear Magnetic Resonance Spectroscopy. Academic Press: San Diego, 1988.

37. Qiu, X.; Moe, N. E.; Ediger, M. D.; Fetters, L. J. Local and Global Dynamics of Atactic Polypropylene by Multiple Field C13 Nuclear Magnetic Resonance. J. Chem. Phys. 2000, 113, 2918-2926.

38. Moe, N. E.; Qiu, X.; Ediger, M. D. C13 NMR Studies of Segmental Dynamics of Atactic Polypropylene Melts. Macromolecules 2000, 33, 2145-2152.

39. Lipari, G.; Szabo, A. Model-Free Approach to the Interpretation of NMR Relaxation in Macromolecules. 1. Theory and Range of Validity. J. Am. Chem. Soc. 1982, 104, 4546-4559.

40. Lipari, G.; Szabo, A. Model-Free Approach to the Interpretation of NMR Relaxation in Macromolecules. 2. Analysis of Experimental Results. J. Am. Chem. Soc. 1982, 104, 4559-4570.

41. von Meerwall, E.; Kamat, M. Effect of Residual Field Gradients on Pulse-Gradient NMR Diffusion Measurements. J. Magn. Reson. 1989, 83, 309-323.

42. Price, W. S. Pulsed-Field Gradient Nuclear Magnetic Resonance as a Tool for Studying Translational Diffusion: Part 1. Basic Theory. Concepts Magn. Reson. 1998, 104, 299-336.

43. Johnson, C. S. Diffusion Ordered Nuclear Magnetic Resonance Spectroscopy: Principles and Applications. Prog. NMR Spectrosc. 1999, 34, 203-256.

44. Price, W. S. Pulsed-Field Gradient Nuclear Magnetic Resonance as a Tool for Studying Translational Diffusion: Part II. Experimental Aspects. Concepts Magn. Reson. 1998, 104, 197237.

45. Hakansson, B.; Nyden, M.; Soderman, O. The Influence of Polymer Molecular-Weight Distributions on Pulse Field Gradient NMR Self-Diffusion Coefficients. Colloid Polym. Sci. 2000, 278, 399-405.

46. Hiemenz, P. C. Polymer Chemistry, the Basic Concepts. Marcel Dekker, Inc.: New York, 1984. 
47. Nilsson, M.; Hakansson, B.; Soderman, O.; Topgaard, D. Influence of Polydispersity on the Micellization of Triblock Copolymers Investigated by Pulsed Field Gradient Nuclear Magnetic Resonance. Macromolecules 2007, 40, 8250-8258.

48. Menjoge, A.; Dixon, J.; Brennecke, J. F.; Maginn, E. J.; Vasenkov, S. Influence of Water on Diffusion in Imidazolium-Based Ionic Liquids: A Pulsed Field Gradient NMR Study. J. Phys. Chem. B 2009, 113, 6353-6359.

49. Iannacchione, G.; von Meerwall, E. Influence of Polydispersity and Amine-Epoxide Ratio on Molecular Mobility in Epoxy Networks. J. Polym. Sci., Part B: Polym. Phys. 1991, 29, 659-668.

50. von Meerwall, E. D.; Ferguson, R. D. A Fortran Program to Fit Diffusion Models to Field-Gradient Spin Echo Data. Comput. Phys. Commun. 1981, 21, 421-429. 
TOC Graphic

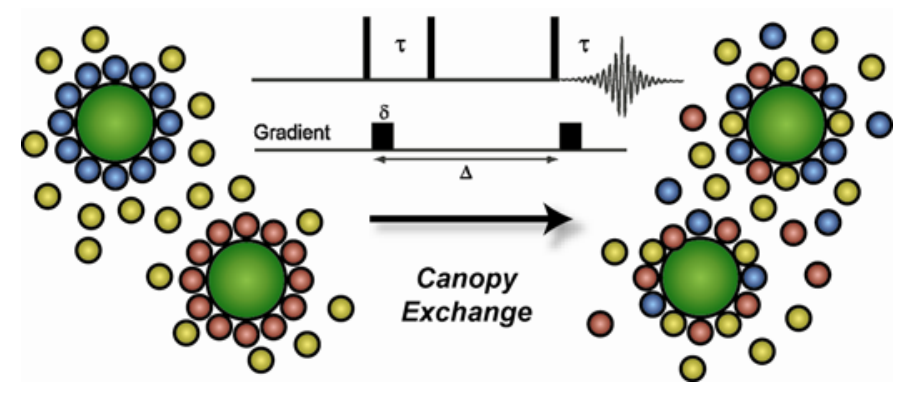

